\title{
THE PROPERTIES OF GEOMETRICALLY MODELLING COMPUTATIONAL SCHEMES FOR BUILDING STRUCTURES
}

\author{
Vladimir POPOV ${ }^{1}$, Eduard KRIKSUNOV ${ }^{2}$, Tatjana GRIGORJEVA ${ }^{3 *}$ \\ ${ }^{1,3}$ Vilnius Gediminas Technical University, Vilnius, Lithuania \\ ${ }^{2}$ SCAD Soft, Kiev, Ukraine
}

Received 10 August 2020; accepted 19 October 2020

\begin{abstract}
For launching a project on a structural object, the calculation of building structures stands as one of the most important stages of project development. In order to correctly analyse structural behaviour, determine the stress-strain state and solve design or inspection problems, the designer is forced to adequately formalize the actual structure turning it into a faultless computational scheme. Virtual testing is one of the main features of the single graphical-information model. Interoperable systems for three-dimensional modelling and analysis, calculation and design ensure smooth data transfer between the physical and computational model. Modern object-modelling techniques and integrated analysis systems allow achieving the defined goal. The article deals with the forms of data exchange, the developmental features of the designed and computational (analysis) BIM model, the integrated design process of CAD/CAE as well as the conversion problems of the physical and computational model.
\end{abstract}

Keywords: BIM, CAD, physical model, analysis model, software integration.

\section{Introduction}

For designing a structural object, an architect and an engineer have to solve many problems such as creating a quality product or designing a project on a building or other structure under strict limited time conditions. The project must guarantee the choice of rational structural solutions thus applying the concept of the architectural shape and space, minimizing the risks of errors and inaccuracies, allowing the most appropriate assessment of real time and resource costs and ensuring the fast and smooth construction process as well as the safe and trouble-free long-term operation of the building structure (Ford et al., 1994; Hoekstra, 2003; Popovas et al., 2003, 2004).

Design and structure fall into a number of smaller areas usually equipped with the specified software. The need for all project participants to acquire a new working method remains one of the key challenges faced trying to fully embrace BIM technology the main premise of which is the cooperation of all stakeholders (project participants) at different stages of the life cycle of the facility. Working together assists project participants in creating quality and reliable information based on the verified decisions made by project parties (Jeong et al., 2009; Hasan et al., 2019; Succar, 2009; Zhu, 2015).
Thus, BIM is a common digital expression of the model based on the interaction standards of project participants, i.e. integration. Modern computer-aided design systems scan and record standard formats used in many typical industries, which ensures the integration of these systems with other prevailing building structure design systems and liaises with structure manufacturers (Miettinen \& Paavola, 2014).

Hardly any software covers all areas of structural design and manufacturing, because no software tool can succeed in all engineering tasks required for creating and manufacturing a product or performing equally well at all stages of designing. As a result, consumers employ the most appropriate and user-friendly software for achieving the specified objectives. Therefore, different software products are mixed and applied in line to operational goals (Migilinskas et al., 2013).

For launching a project on a structural object, the calculation of building structures stands as one of the most important stages of project development. Any structural and many architectural and engineering solutions related to structures must be based on calculations and meet requirements for functionality, strength, reliability and du-

*Corresponding author. E-mail: tatjana.grigorjeva@vilniustech.lt 
rability. In order to correctly analyse structural behaviour, determine the stress-strain state and solve design or inspection problems, the designer is forced to adequately formalize the actual structure turning it into a faultless computational scheme. Crucial decisions have to be made and implemented by the designer within a limited time to help project participants with developing a graphicalinformation model conforming to the BIM methodology (Popov \& Grigorjeva, 2007).

Virtual testing is one of the main features of the single graphical-information model. Interoperable systems for three-dimensional modelling and analysis, calculation and design ensure smooth data transfer between the physical and computational model. Modern object-modelling techniques and integrated analysis systems allow achieving the set goal. Nevertheless, it has historically been the case that computer graphics systems and structural computing and analysis programs have long developed in parallel as independent directions, but recent automated design systems (BIM) have introduced different levels of integration between the graphical environment and computing and analysis programs. The integration level is the most important basis for developing the single graphical-information model (Popov \& Grigorjeva, 2010).

The article examines the forms of data exchange, the developmental features of the designed and computational (analysis) BIM model, the integrated CAD/CAE design process as well as the conversion problems of the physical and computational model.

\section{The forms of data exchange}

The extensive application of three-dimensional graphical information modelling software for simulating buildings and structures has created preconditions for using the above introduced models in the field of developing computational schemes.

The software platforms such as ALLPLAN, Revit, Tekla Structures, etc. provide the ability to directly transfer data to computing and analysis systems while forming computational schemas. In the cases when other programs are used for structural strength analysis, the initial data are generated by converting the original model applying specific programs - converters. The current most common technologies used for computational schemes are based on the information model and include:

1. API (Application Programming Interface) used for developing convertors that allow the conversion of models into input formats for specific structural calculation and analysis applications;

2. converting an object model presented in the format of the international standard IFC (Industry Foundation Classes) into computational schemes;

3. using a specified format, for example, SDNF (Steel Detailing Neutral Format - a neutral format describing the 3D models of metal structures) applied by Tekla Structures or CIS/2 (CIMSteel standard) adopted by the American Institute of Steel Con- struction (AISC) as a format of data exchange software for designing steel structures;

4. the direct transfer of a 3D model for a solid body from a modelling system to a computing environment for further use in the analysed model; despite the positive experience of using APIs, changes in the internal formats of modelling programs considering format enhancement cause variations in API and thus constantly adjust converters.

The IFC format has been developed as an open data exchange standard and is defined as one of the basic and most universal standards for presenting BIM information. However, the lack of information about the elements of a particular assortment of the rolled metal products seems to be a significant shortage of this format employing it in line to exchanging data with structural calculation and analysis programs. The performed calculation and the selection of element cross-sections provide that information about new-cross sections is sent back from the calculation program to BIM in the form of a parameter element. In addition, IFC does not ensure the unambiguous interpretation of information in various programs, which is due to the fact that modelling the same object results in the different shapes of that particular object (Oraskari \& Törmä, 2015; Kouhestani \& Nik-Bakht, 2020).

The use of specific formats like SDNF and CIS/2 allows for the most comprehensive transmission of model information. Nevertheless, employing these formats is limited to their applicability to certain types of objects (in this case, steel structures) (El-Diraby et al., 2017).

The obtained converted scheme typically consists of a set of structural elements each corresponding to the specific element of the load-bearing structure of the original model. The elements may involve columns, ceilings, beams, walls, etc. Some design systems refer to such models as to "computational" while other systems treat them as "analytical". The characteristic feature of the models is that every detail of the model is present in the erected building or structure.

Software for structural calculations and analysis refer to the concepts of "a computational scheme" and "a calculation model" as to the synonymous, and the models composed of structures are conditionally considered to be "structural".

\section{Structural models}

The information model for building structures, including all attributes, the so-called physical model, is the key deliverable of working on the BIM paradigm and occupies the central place in the group of BIM uses. The model is known by different names at the varying stages of project development, embracing Inventory Model, BIM Model, As-built Model, Record Model, Asset Model, and provides access to all standard project presentations like visualization, drawings, quantity sheets, specifications, collision reports and other various analysis cases and simulations (Eastman et al., 2011; Tang et al., 2010; Watson, 2011). 
Although the physical model for the building acts as the result of interdisciplinary collaboration, it largely consists of an architectural-structural model that shows the overall structure of the building, identifies the interaction between space, shape and function as well as combines many elements characteristic of all disciplines of the project. The main feature of the physical model is the strict coincidence of the geometric shapes of the model with the elements of the actual would-be structure built and installed pursuant to this particular model. A structural model is a part of the physical model that combines only structural elements and covers all load-bearing structures of the building designed to ensure the strength and stability of the building thus taking over and transferring loads and impacts to the foundation of the building. The physical creation of the model is the main technological and functional object of all so-called "BIM applications".
Structural models are characterized by the actual shape and size of the elements (Figures 1,2). Two-dimensional structures such as walls or panels are of a free-shape and have openings and characteristics, including data on the material and thickness. The model attachment point may provide information on the median plane eccentricity with respect to the attachment point. Linear elements (columns, beams) are described by the material, shape and size of the cross-section and are given a possibility of indicating the eccentricity of the longitudinal axis in terms of the point of attachment.

Structural models are intermediate models between BIM and the computational scheme developed conforming to the rules that comply with the calculation method used and the specific calculation and analysis program. The possibility of developing, importing and exporting structural models is specified in almost every program for calculating and analysing building structures.

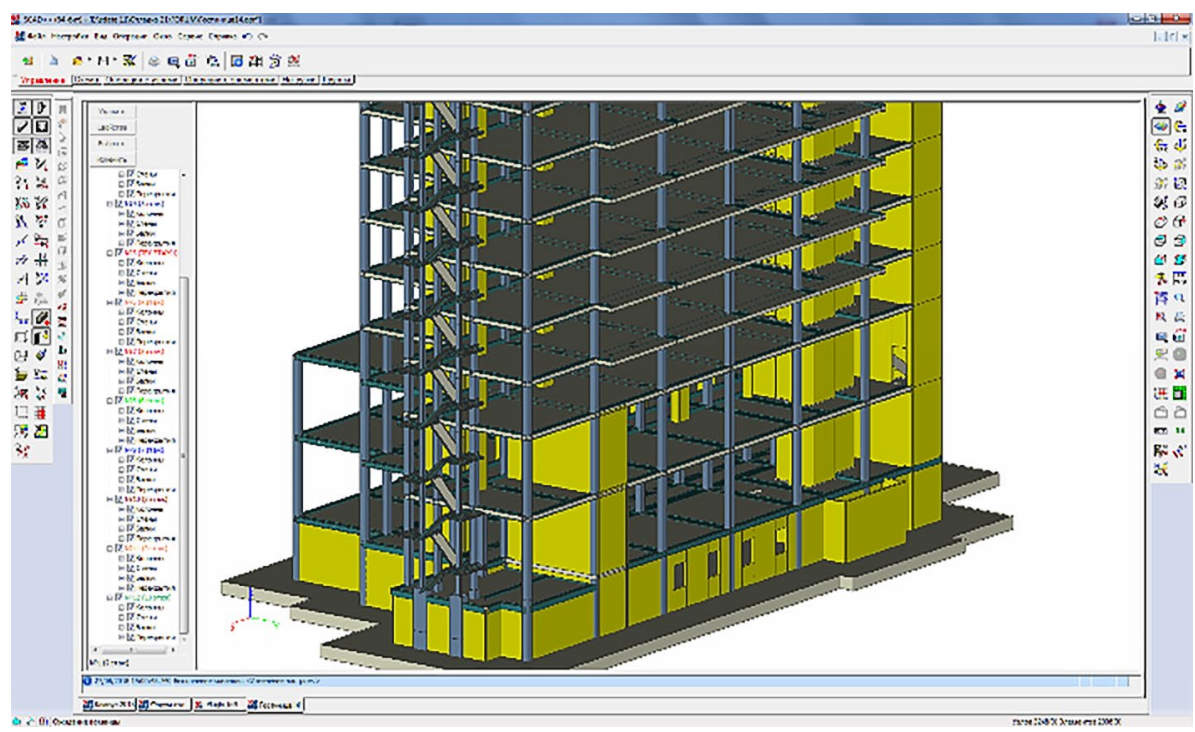

Figure 1. A fragment of the model for a building made of reinforced concrete structures

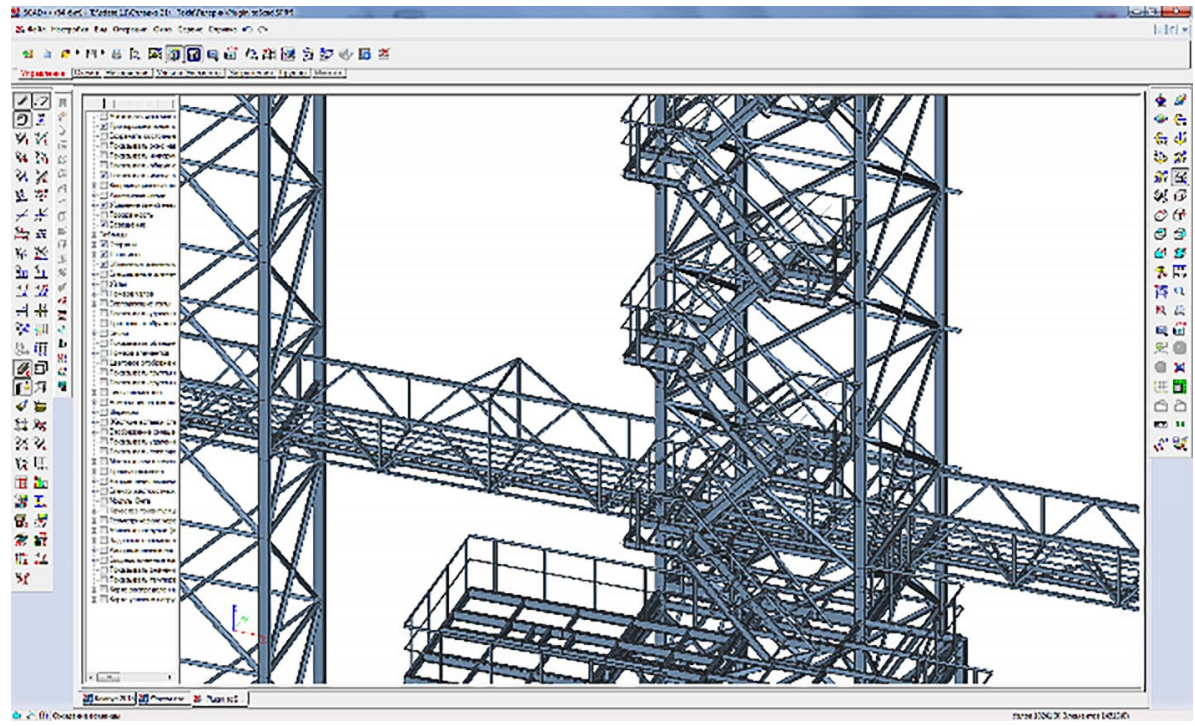

Figure 2. A fragment of the model for a building made of metal structures 


\section{The calculated (analysis) BIM model}

As for calculations, many modern widely used structural calculation and analysis systems use the finite element method (FEM), i.e. the structural model reflected by the set of structures is converted into the FEM computational scheme before calculation is done.

The analysis model is the so called "computational scheme". The analysis model does not necessarily have to accurately reproduce the geometric shapes of the object, but rather, it is mathematical abstraction designed to describe the mechanical properties of the structure as accurately as possible and to reliably model (predict) abstraction behaviour under load conditions. In order to correctly simulate structural behaviour, to analyse the stress-strain state of the structure and to solve the problems of strength testing or design, it is necessary to adequately formalize the actual structure turning it into the ideal analysis model (computational scheme).

The specificity of the analysis model is subject to the analysis method and the tools of mathematical implementation. The finite element method is employed for modelling the body or its area that produces a solution (analysis model) and is divided by simple geometric shapes applying a discrete (finite) element network.

Finite elements can be one-dimensional (1D) rods, flat or shell-shaped two-dimensional (2D) and spatial/ volumetric, i.e. three-dimensional (3D). One-dimensional (1D) rods are designed to model structures having one dimension significantly larger than the others two. Flat or shell-shaped two-dimensional elements are created to model structures having two dimensions significantly larger than the third one. 3D spatial elements are designed to model structures the dimensions of which are of a size of the single row. The same structure (even clearly linear) present in the analysis model is approximated using the finite elements of a similar type, i.e. rod-shaped, shellshaped, volumetric, or a combination of those, for example, bar- and shell-shaped, volumetric and bar-shaped, volumetric and shell-shaped.

Modern modelling systems form an information model comprising a description of structures, information about connections, loads, boundary conditions of elements, etc. Theoretically, transition from the structural model to the computational schema is considered a simple data conversion operation. In addition, based on this assumption, the results of the analysis and further selection of reinforcement or steel structure elements could easily be transferred to the initial model and implemented in the project. Unfortunately, this simple and seemingly easy-toimplement data processing scheme turns out to be purely theoretical in practice.

\section{The calculated (analysis) BIM model}

The ideal case of the design process based on the concept of integrated graphical-information (BIM) and analysis (CAE) models covers the following main technological stages (Figure 3):

a. the BIM (CAD) system creates the BIM model (physical model for the structure) having all required characteristics of the actual structure, including the position of elements in space, the geometry of elements, material characteristics;

b. the model is transmitted to the structural analysis and design system (CAE) conducting necessary operations that allow transforming the physical model to the analytical one;

c. the CAE system describes the boundary conditions of the analysis model and assigns the required design parameters;

d. model (virtual testing) is analysed to assess structural behaviour, to check the existing or find the optimal structural solution;

e. design parameters proposed by the CAE system (cross-sections, reinforcement, fasteners, etc.) are selected and assigned to structural elements and their assemblies;

f. the obtained results are transmitted back to the BIM (CAD) system;

g. the CAD system rearranges and elaborates the geometry of elements and their connecting nodes taking into account the findings of the conducted analysis;

h. a standard set of technical documentation is issued.

An alternative scenarios of workflow design is also possible in the case when the analysis model is developed separately from the physical model for the building and the results obtained following all required analysis steps are assigned to the CAD (BIM) model (Figure 4). However, this is not an example of an integrated system.

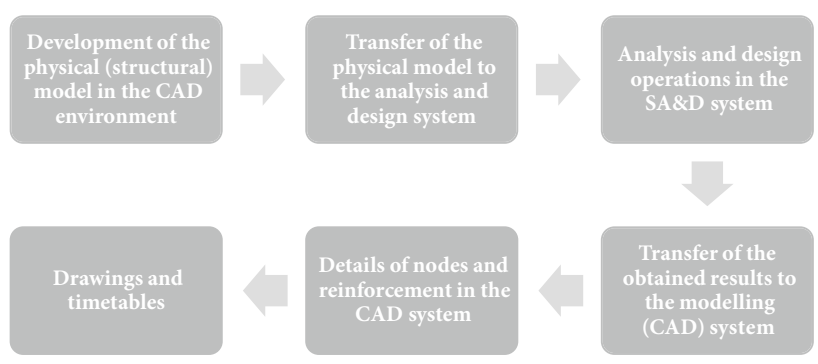

Figure 3. The design process based on the concept of integrated graphical-information (BIM) and analysis (CAE) models

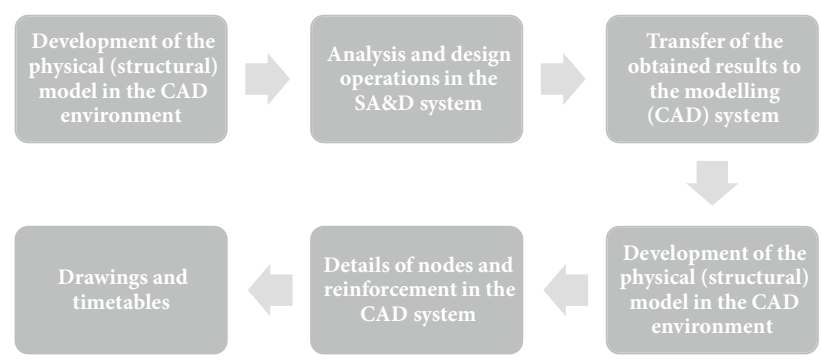

Figure 4. An alternative design process showing the analysis model developed separately from the physical building model 
Figure 4 above provides the ideal technological scheme. When it comes to the integration of modelling and analysis systems, plenty of specific aspects are encountered thus emphasizing two most important points - the principles of building the analysis model and the way of data exchange (integration level).

\section{The problems of the physical and computational model}

Some of the problems arising in the process of forming the computational scheme based on BIM are discussed below. In this case, the focus is shifted on the issues related to the geometry of computational schemes.

From a design point of view, the main idea of BIM is integrating parametric models for the designed object into a single system, including the possibilities of their interaction and mutual influence. This means that for whatever reason, changes in one of the models are automatically reflected throughout the complete system. Yet at this stage, actual difficulties are related to the computational scheme devised for a particular computational and analytical program and having certain characteristics such as:

- to comply with data submission rules adopted in the application used;

- to reflect the vision of the designer for structural behaviour under various loads and effects;

- to consider different conditions for the interaction between the structure and the environment;

- to take into account the uncertainty of many initial parameters affecting structural behaviour etc.

The list above shows that serious projects are unlikely to be limited to a single computational scheme. For example, almost two dozen design models were used for analysing the stress-strain state of a new sarcophagus structure at Unit 4 of the Chernobyl Nuclear Power Plant. Approximately the same number of models were employed for analysing the relatively large structure of a 25-story monolithic reinforced concrete house. Such alternatives are hardly accepted to be obtained automatically from the BIM model. All these versions of models do not initiate automatic architectural (technological) and related model changes in calculation programs.

For analysing the stress-strains state of complex construction objects, including high-rise buildings, changes in geometry during the construction process must be considered. As a result, certain hardly implemented modelling rules need to be followed, which means that a computational engineer must be involved. Similar problems have to be addressed to analysing progressive disintegration, exposure to extreme effects, etc. The quality of calculation and analysis, as a rule, is also subject to the correct assessment of conditions for the interaction of the structure with substrate.

Plenty of the initial parameters for the model are barely determined with the required accuracy. This is particularly true of the condition of emergency or reconstructed struc- tures and their connection units. In this case, the processes of calculating several versions changing the characteristics of the elements in the range of possible values and making a design decision based on the results of such multivariate calculation are required.

The isogeometric method implemented in the FEA program MicroFE is one of the successful examples of automatically creating a calculation scheme based on the "solid-state" structural model, which allows estimating the actual sizes of structural elements and the rules of their interaction. The proposed method can be used for estimating buildings made of monolithic reinforced concrete. At the same time, the isogeometric method does not exclude the analysis of structural behaviour considering changes in geometry during installation, multivariate analysis, the use of multiple models, etc.

The analysis of a model fragment (Figure 6) of a rather typical object (Figure 5) shows that many connecting elements and nodes of structural elements hardly affect the load-bearing capacity of the structure. The formation of the structural model is not included in the computational scheme, which is ensured by the advanced filtering system available in most modelling applications.

Even the processes of calculating load-bearing capacity and selecting cross-sections to update element parameters in the BIM model do not guarantee that many of these elements will remain in place or maintain their dimensions. This applies particularly to steel structure elements. Thus, it is likely that a significant part of the project will have to be redesigned. The made calculations also show the problems of structural changes in the nodes of loadbearing structures. In the case node calculations are done along with the analysis of bearing capacity, the nodes of the modelling system must be parametric objects and automatically "adapted" to the new cross-sections of the connected elements.

The above statements show that CAE (computer engineering) processes related to load capacity calculations occupy a special place in BIM technology. The formation of computational models for load-bearing structures should occur before the processes of detailed design. At the same time, technology that employs the computational scheme created using the tools of calculation programs cannot be ruled out regardless of the physical model for the designed object.

To sum up, the established practice of forming the geometry of computational schemes based on DWG/DXF data obtained working in one of the graphical editors, for example "AutoCAD", should be mentioned. Computational schemes are launched with reference to 2D drawings, including the object itself, dimensional lines, specifications, comments and other elements of drawing design. If a designer has taken care of dividing information into layers, separating the actual geometry of the object is straightforward. The resulting graphical representation consisting of a number of points and the lines connecting them is accepted as the basis for compiling the computational scheme. 


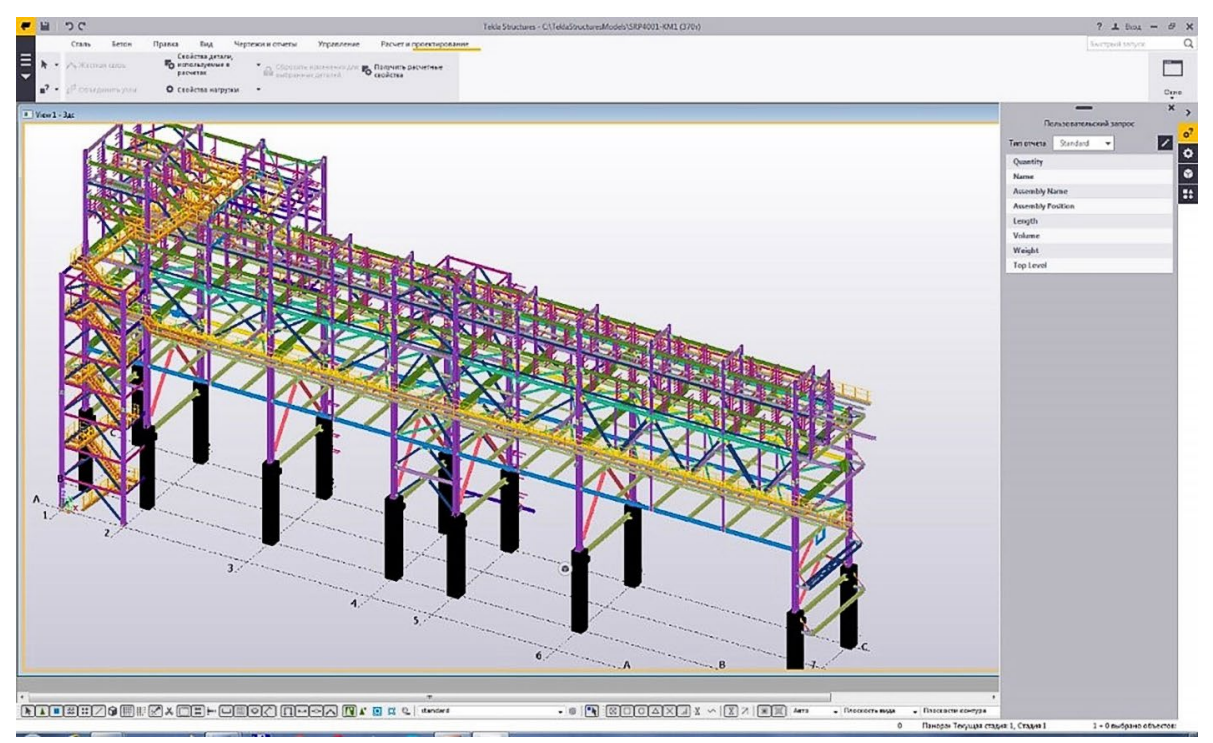

Figure 5. A physical model for the structure applying Tekla Structures software

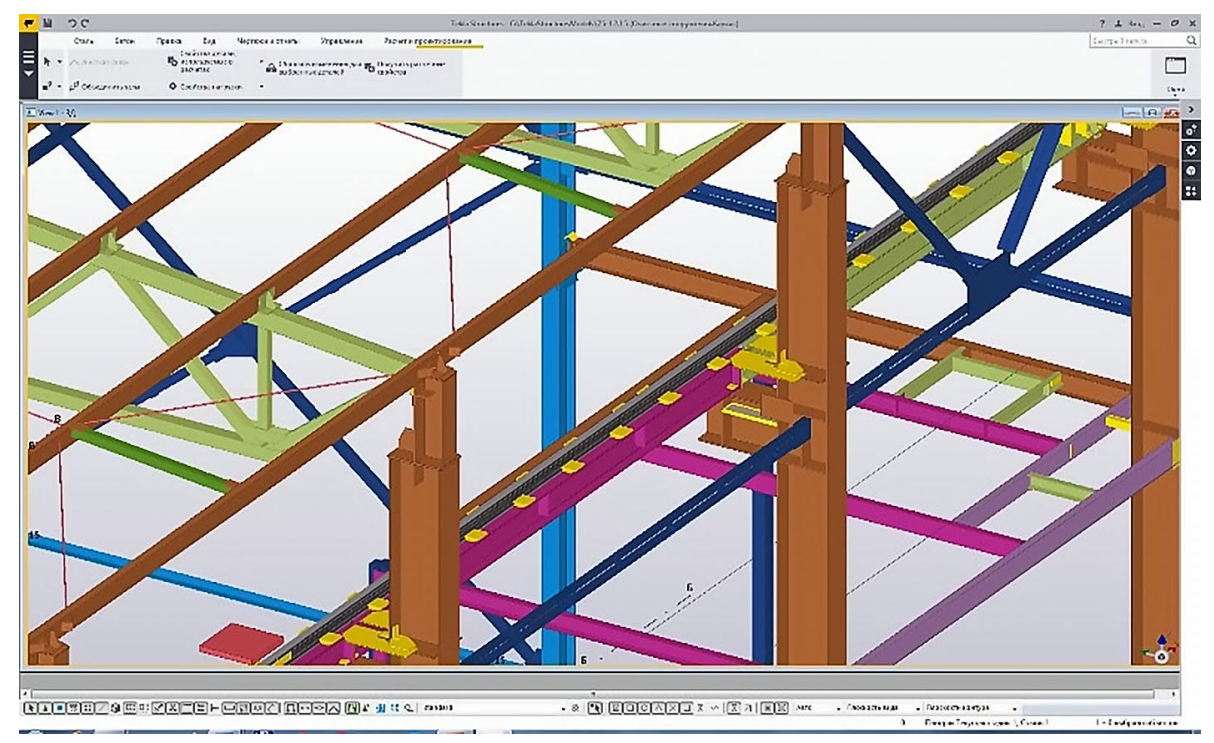

Figure 6. A fragment of the physical model for the structure applying Tekla Structures software

\section{Conclusions}

The employment of modern automated design systems with the capabilities of integration between the graphical (BIM) platform and the computing system provides that the development of the computational model for building structures can be twofold. One of the methods involves the conversion of the virtual graphical-information model for a construction object to the rod or shell-shaped computational model or a combination of the rod-shaped and shell-shaped models during the transfer to the calculation system. The other technique embraces the transfer of the virtual graphical-information model for the construction object as a physical model thus maintaining the geometrical and physical characteristics of the structure.

In order to implement the basic principle of BIM, i.e. to efficiently integrate software, the project of the struc- tural part requires the ability to transfer data from the structural physical model (developed using specified CAD modelling tools) to the analysis model (created employing the indicated CAE analysis tools). A different nature (ideology) of the above introduced tools and applied methods and technologies present a number of problems in the process of converting the design (BIM) model to the analysis model.

Two main problems, including one of technical and the other - of conceptual nature, are encountered at the model conversion stage.

A different topology of the models is conceptual, and therefore the BIM-matched physical model is inappropriate for calculation (analysis) due to difference in the essential roles. On the one hand, the exact reproduction 
of the shape, whereas on the other - mathematical abstraction is required to simulate mechanical behaviour. A technical problem covers conversion from the structural (physical) to analysis (computational) model and is difficult to be automatically performed due to shortage of data or conversion (transmission) problems, i.e. information (primarily graphical) cannot be transmitted without loss and distortion, and data (attribute) are not present in the CAD model (system simply does not generate such information).

The main problem of BE model compatibility is addressed to many specific mathematical nuances in the calculation of structures the implementation of which depends on the BEM environment where calculation is made.

\section{References}

Eastman, C. M., Sacks, R., Liston, K., \& Teicholz, P. (2011). BIM handbook - a guide to Building Information Modeling for owners, managers, designers, engineers, and contractors ( $\left.2^{\text {nd }} \mathrm{ed}.\right)$. John Wiley \& Sons.

El-Diraby, T., Krijnen, T., \& Papagelis, M. (2017). BIM-based collaborative design and socio-technical analytics of green buildings. Automation in Construction, 82, 59-74. https://doi.org/10.1016/j.autcon.2017.06.004

Ford, S., Aouad, G., Brandon, P., Brown, F., Child, T., Cooper, G., Kirkham, J., Oxman, R., \& Young, B. (1994). The object oriented modelling of building design concepts. Building and Environment, 29(4), 411-419. https://doi.org/10.1016/0360-1323(94)90001-9

Hasan, A. M. M., Torky, A. A., \& Rashed, Y. F. (2019). Geometrically accurate structural analysis models in BIM-centered software. Automation in Construction, 104, 299-321. https://doi.org/10.1016/j.autcon.2019.04.022

Hoekstra, J. (2003). Big Buzz for BIM. Architecture, 92(7), 79-82.

Jeong, Y.-S., Sacks, R., Kaner, I., \& Eastman, C. M. (2009). Benchmark tests for BIM data exchanges of precast concrete. Automation in Construction, 18(4), 469-484. https://doi.org/10.1016/j.autcon.2008.11.001

Kouhestani, S., \& Nik-Bakht, M. (2020). IFC-based process mining for design authoring. Automation in Construction, 112, 103069. https://doi.org/10.1016/j.autcon.2019.103069

Miettinen, R., \& Paavola, S. (2014). Beyond the BIM utopia: Approaches to the development and implementation of building information modeling. Automation in Construction, 43, 84-91. https://doi.org/10.1016/j.autcon.2014.03.009

Migilinskas, D., Popov, V., Juocevičius, V., \& Ustinovičius, L. (2013). The benefits, obstacles and problems of practical BIM implementation. Procedia Engineering, 57, 767-774. https://doi.org/10.1016/j.proeng.2013.04.097

Oraskari, J., \& Törmä, S. (2015). RDF-based signature algorithms for computing differences of IFC models. Automation in Construction, 57, 213-221. https://doi.org/10.1016/j.autcon.2015.05.008

Popovas, V., Jarmolajevas, A., \& Grigorjeva, T. (2003). Šiuolaiknės automatizuoto projektavimo sistemos. Nauja statyba, 6-7, 26-29, 40-41 (in Lithuanian).

Popovas, V., Ustinovičius, L., \& Mikalauskas, S. (2004, May). Technique for computer aided evaluation of economic indicators of a construction project. In The $8^{\text {th }}$ International Conference "Modern Building Materials, Structures and Tech- niques": Selected papers (Vol. 1., pp. 242-248). Vilnius, Lithuania. Technika.

Popov, V., \& Grigorjeva, T. (2007). Statybiniu konstrukciju projektavimas taikant integruotas kompiuterinio projektavimo sistemas. Pažangioji statyba: pranešimų medžiaga (pp. 30-39). Technologija (in Lithuanian).

Popov, V., \& Grigorjeva, T. (2010). Integrated automated design of building structures. Engineering Structures and Technologies, 2(1), 31-37. https://doi.org/10.3846/skt.2010.04

Succar, B. (2009). Building information modelling framework: A research and delivery foundation for industry stakeholders. Automation in Construction, 18(3), 357-375. https://doi.org/10.1016/j.autcon.2008.10.003

Tang, P., Akinci, B., Lipman, R., Lytle, A., \& Huber, D. (2010). Automatic reconstruction of as-built building information models from laser-scanned point clouds: a review of related techniques. Automation in Construction, 19(7), 829-843. https://doi.org/10.1016/j.autcon.2010.06.007

Zhu, Y. (2015). Ontology to support multi-objective integrated analyses for sustainable construction: A conceptual framework. In R. R. A. Issa \& I. Mutis (Eds.), Ontology in the AEC industry: A decade of research and development in architecture, engineering, and construction (pp. 73-95). ASCE. https://doi.org/10.1061/9780784413906.ch04

Watson, A. (2011). Digital buildings - challenges and opportunities. Advanced Engineering Informatics, 25(4), 573-581. https://doi.org/10.1016/j.aei.2011.07.003 